\title{
Reviewing scientific manuscripts: A comprehensive guide for peer reviewers
}

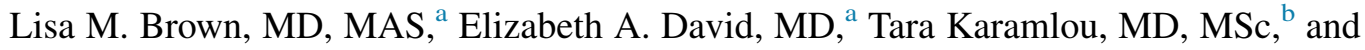 \\ Katie S. Nason, MD, MPH
}

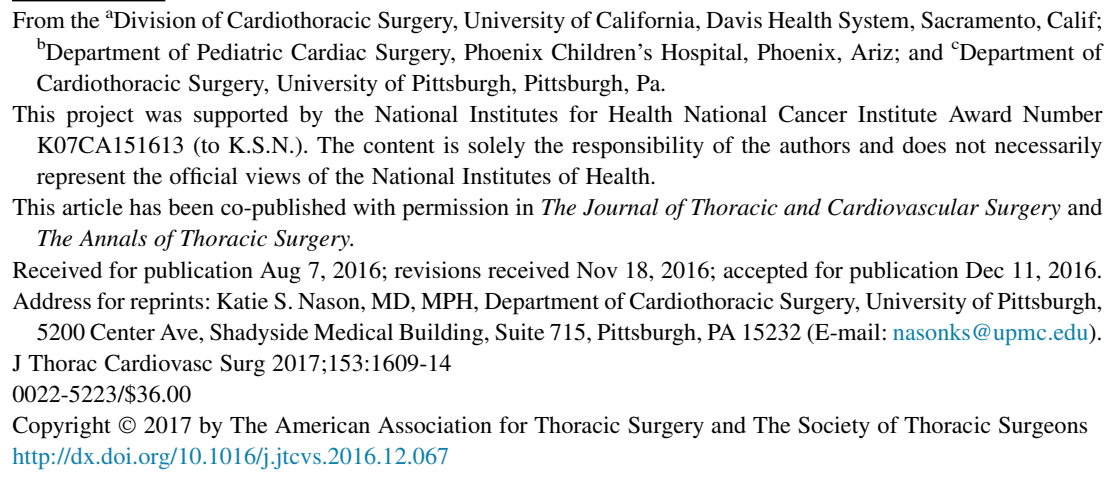

Feature Editor's Note-There are so many things we do as part of our profession that are never purposely taught during our training. These activities simply are not part of our curriculum. Yet there is frequently an assumption that if we excel at performing surgery, all these other tasks will also be performed at a high level. Things like billing, preparing talks, giving feedback, leadership skills, and reviewing a paper are just a few examples in which we assume competence is extrapolated from our achievement of competence in clinical care.

Alas, the truth is that these activities are skills, and like any skill they must be mastered independently. Being a skillful surgeon does not automatically grant the same level of skill in other domains. Dr Nason and colleagues have directly addressed one of these examples, the ability to perform a meaningful peer review. Although this is not a systematic review of the literature (very little if any literature exists), it does provide a clear and complete framework on what constitutes, in these authors' minds, an efficient and consequential review. As an editor, their description, if even adhered to by only most reviewers, would greatly improve the quality of peer review.

If we, as a profession, are intent on advancing our field and remaining relevant, then high-quality peer review of our scientific work is mandatory. Dr Nason and colleagues have provided a framework for the process, but to truly be effective, we must engage broad representation of our profession. Providing peer review is not the exclusive purview of the academics who provide the manuscripts. To truly reflect the knowledge within our profession, the peer-review process must include a generalizable representation of those who engage in our profession. This work by Dr Nason and colleagues provides the tools to achieve that end.

Ara Vaporciyan, MD

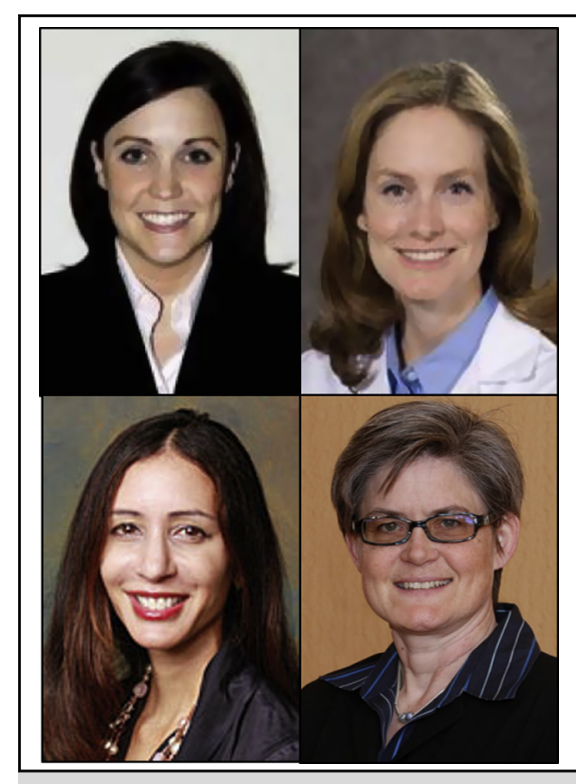

Lisa M. Brown, MD, MAS, Elizabeth A. David, MD, Tara Karamlou, MD, MSc, and Katie S. Nason, MD, MPH (top left to bottom right).

\section{Central Message}

By understanding and implementing the elements of reviewing scientific manuscripts, peer reviewers will contribute to accurate and impactful published literature.

See Editorial Commentary page 1615.

Journal editors rely on peer review from physicians, biomedical researchers, and biostatisticians to critically examine study aims, design, and methodology, and ensure that analyses and conclusions are accurate before manuscript publication. Given the importance of this process and clinician reliance on the published literature to guide clinical practice, we sought to provide peer reviewers guidance and a rubric for performing optimal reviews.

\section{THE EDITORIAL PROCESS}

Following submission, the journal's editor selects peer reviewers, e-mails invitations, and uses the feedback to guide publication decisions. Journals set deadlines for reviewers to (1) respond to the invitation and (2) submit the review. A prompt response from reviewers is vital to moving the process forward. If the reviewer has a conflict 
of interest, she or he should decline the review and have the opportunity to state the reason for doing so. The peer reviewer is expected to make recommendations to the editor, based on his or her comprehensive assessment of the manuscript, as to acceptability for publication. ${ }^{1-3} \mathrm{~A}$ thorough review can take approximately 3 hours, but varies with experience. ${ }^{1,3}$ Exceptional reviews provide the editor and author(s) clear, concise, insightful, and constructive feedback, which accurately reflect manuscript strengths and weaknesses. They also provide specific recommendations for revisions that should significantly improve the manuscript. The following provides a step-by-step approach to optimal review of scientific manuscripts (Table 1).

\section{TITLE PAGE}

The title, author list, institutional affiliations, prior presentation of the data, and corresponding author are universal components of the title page and should accurately and truthfully represent the contributions to the manuscript and the responsible parties. The title is an often overlooked but critical feature of each manuscript. An informative, compelling title will entice readers into reading the paper whereas a bland or nondescript title may cause readers to skip over an otherwise excellent paper. The reviewer may guide authors to revise the title to descriptively capture the essence of the paper. Each journal has instructions for authors that provide guidelines regarding the required elements for the title page, including the number of words or characters allowed in the title and other details. These journal-specific instructions should be familiar to the reviewer and followed by the authors. Additional information may be requested on the title page, including statements of author contributions, conflicts of interest, word count, key words, acknowledgment of funding sources, and central or perspective messages. If these are incorrectly presented or missing, instructions to the authors to correct the errors will reduce time to publication by correcting them early in the revision process.

\footnotetext{
ABSTRACT

The abstract provides the authors with an opportunity to summarize the objectives, methods, results, and conclusions for the journal readers. It is oftentimes the first, and perhaps only, section of the manuscript that will be read, as it is typically freely available through reference databases. The abstract should provide a clear statement of the study objectives, which must match what is stated in the introduction and other summary statements regarding the study. This is oftentimes not the case, and the astute reviewer will identify the discrepancy for the authors to correct. Although brief in length, the abstract methods must define the study group, stratification variables if any, and provide a general overview of the analysis plan. The results should provide data that directly address the stated
}

TABLE 1. Key elements of a scientific manuscript

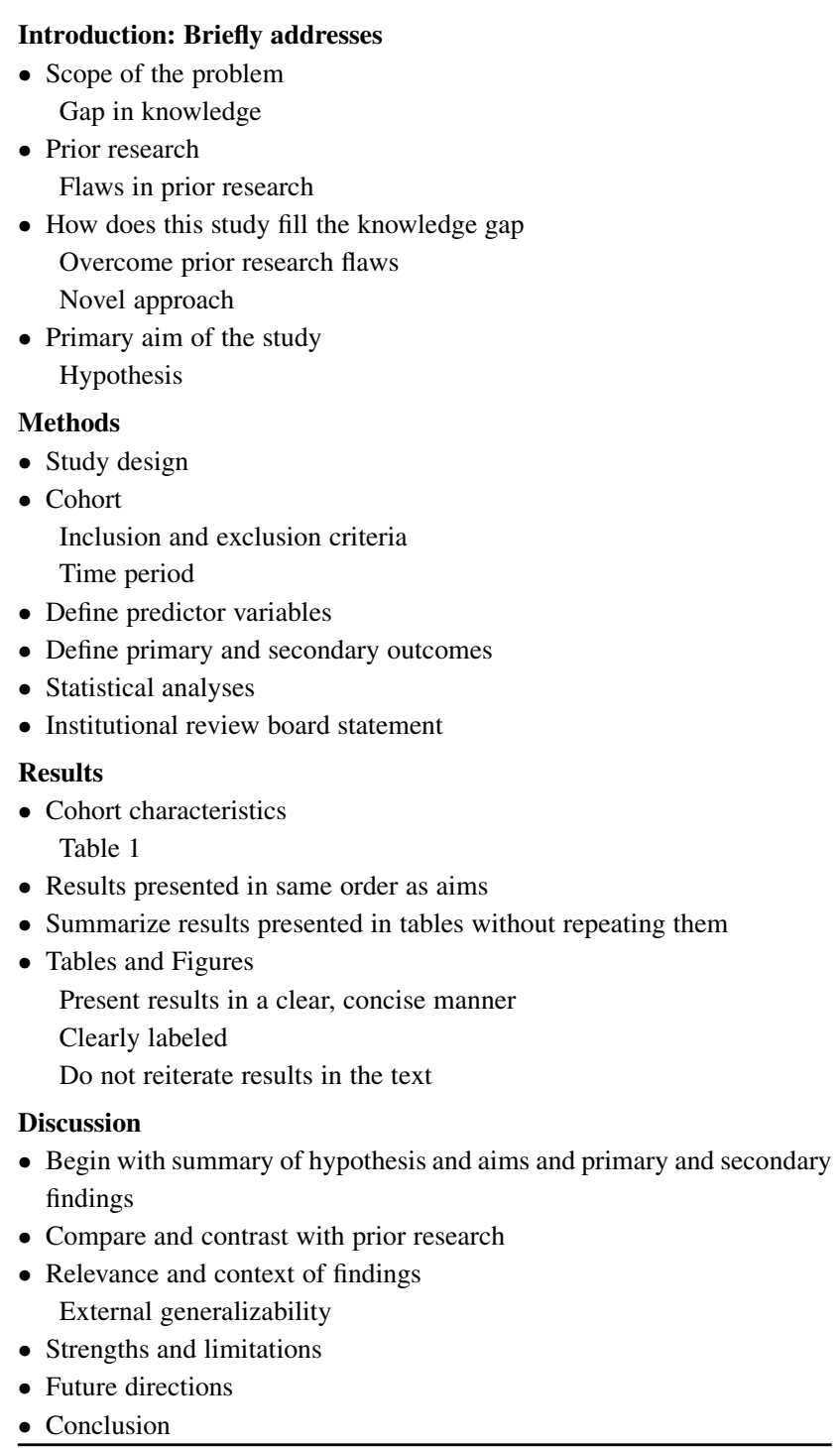

objectives and support the abstract conclusions. Conclusions that are not directly supported by the data provided in the abstract results should not be included in the abstract; these conclusions belong in the manuscript discussion or the appropriate data added to the abstract results. It is often the case that the abstract is excessively wordy without added meaning. Authors often reply to reviewers that the word count restricts the information that can be provided, but this typically can be addressed through language simplification and removal of extraneous words. If this is the case, the reviewer will advise the authors to revise accordingly.

\section{INTRODUCTION}

The introduction succinctly defines the scope of the problem and justification for further investigation. ${ }^{4}$ It should be 
no more than 2 to 3 paragraphs. Unfortunately, many authors attempt to "set the stage" with extraneous information not germane to the study hypothesis or aims, such as global statements about topics (epidemiology, survival, treatment) that are relevant to the disease but not under investigation in the current study. When this is encountered, valuable reviews provide authors constructive feedback to revise and limit the introduction to a brief statement on the scope, importance, and context of the problem relevant to and congruent with the specific research question. The strengths and limitations of prior relevant studies are briefly introduced, but in-depth critique of them is reserved for the discussion section. The final paragraph of the introduction is a clear statement of the study aim(s) and hypothesis. For studies focusing on clinical questions, the aim should be testable and clearly identify the patient, population, or problem; the intervention, prognostic factor, or exposure of interest; the comparison groups or alternative intervention; and the outcome to be evaluated. Often when the methods, results, and discussion are confusing, it is because the study lacks clearly stated and testable aim(s).

\section{METHODS}

The methods describe how the study aim(s) were tested and include the elements described in the following paragraphs. Effective reviews provide authors feedback when these requirements are not met, including sufficient information for each element such that independent investigators could replicate the study.

1. Type of research study and study participants: This includes type of study (Table 2), population, study setting, detailed inclusion and exclusion criteria, and study time frame/dates. ${ }^{5}$ Critical evaluation of the appropriateness of the study design is a central feature of an outstanding review. It is important to query whether the design allows for the question(s) to be answered and whether the numbers of patients are sufficient. The reviewer will assess whether the inclusion and exclusion criteria are justified and allow for the study question to be evaluated.

2. Reporting guidelines: Reporting guidelines by study type are available and useful to both authors and reviewers as a checklist to ensure that all key elements of a study are included in the manuscript ${ }^{6}$ (Table 3 ). For example, all clinical trials must have an accompanying CONSORT (Consolidated Standards of Reporting Trials) flow diagram that demonstrates the progress through the phases of a randomized clinical trial involving 2 groups. ${ }^{7}$ These phases include enrollment, intervention allocation, follow-up, and data-analysis. The reviewer will assess these diagrams to make sure that the published protocol has not significantly deviated from the intended trial protocol.
3. Data source: The data source and methods for data collection should be succinctly but accurately described and referenced, including whether the data were prospectively versus retrospectively collected, who collected the data, and whether the data abstractors were blind to the study question or intervention.

4. Predictor variables: Any novel, complex, or key study predictor variables should be defined in detail, including how they were calculated and/or measured. As with the study outcomes (see the next paragraph), the predictor variables should be defined consistent with the published literature or justification provided for an alternate definition.

5. Outcomes: The primary and secondary study outcome(s) should be stated and clearly defined, consistent with the published literature. Examples of outcomes often inadequately defined include survival outcomes; studies will often provide an "end date" for the study outcome that is not linked to known patient status. This is incorrect and should not be accepted. For overall survival, the "end date" is patient-specific: it is the date of death or the last date the patient was known to be alive. Unless the investigator contacted all patients on the specified study "end date" and confirmed alive status (highly unlikely), a thorough review will identify the data definition error and recommend that the data be reanalyzed, censoring alive patients on the date that they were confirmed alive. Similar problems exist for disease-free survival analysis. Finally, another common error encountered occurs when analyzing outcomes in which the endpoint may not be known precisely (for example, freedom from structural valve deterioration [SVD]). Unless the definition of SVD is defined by echocardiographic criteria (gradients or degree of regurgitation) and the date of the echocardiogram is ascertained, an actuarial analysis may be flawed. Although a reviewer may not know how to correct each problem, alerting the editor to such issues will facilitate additional input from reviewers with focused expertise.

6. Statistical analyses: Outstanding reviewers understand that statistical tests performed should be appropriate for the study question and the data being analyzed. ${ }^{8}$ As with variable definitions, sufficient detail is required such that an independent investigator could replicate the analysis. Reproducibility of methodology and results is an area of priority for the National Institutes of Health, and reviewers should ensure that the descriptions provided are adequate and feasible to account for the results. ${ }^{9}$ Moreover, an astute reviewer will recognize the importance of properly used descriptive statistics that respect the normality of reported variables. Examples in which details are often omitted include methods for developing multivariable regression 
TABLE 2. Types of clinical research studies

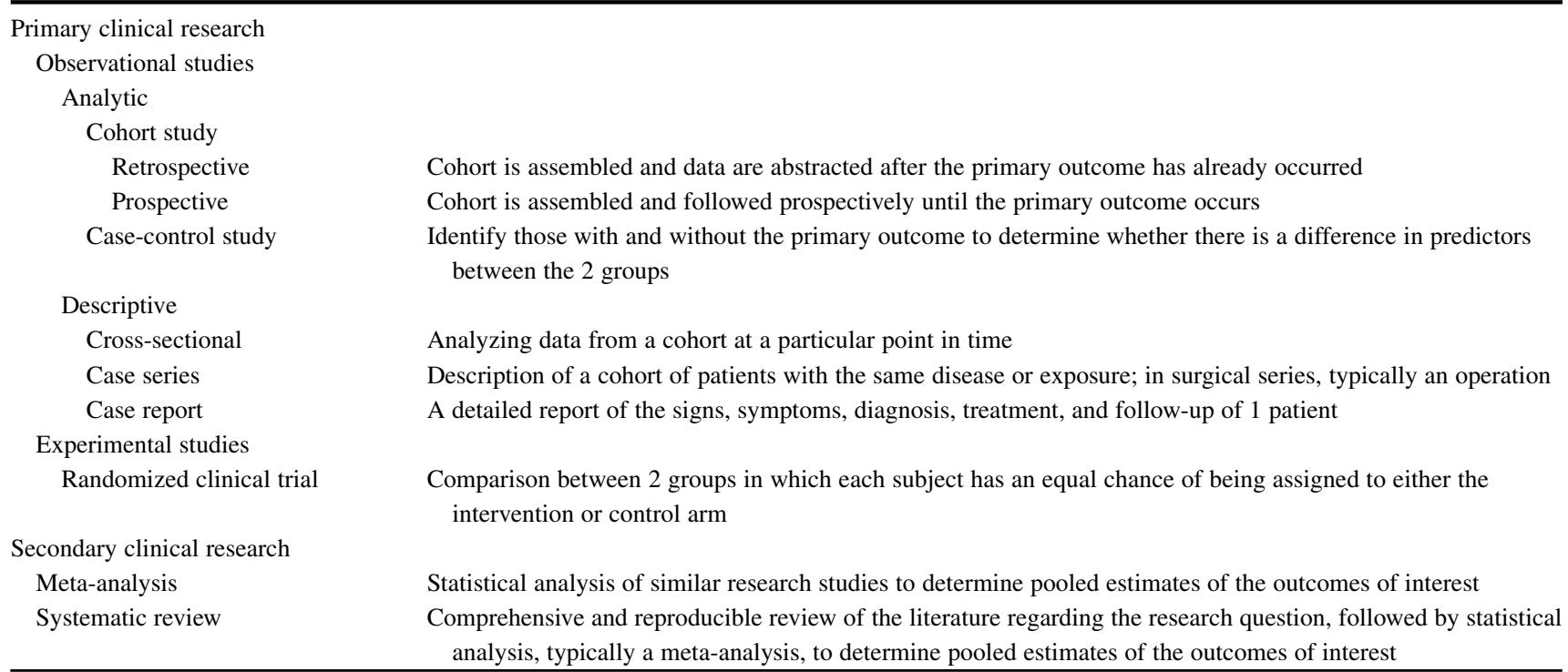

models, ${ }^{10}$ assignment of propensity scores or other matching algorithms, and handling of missing data, among others. By approaching the review process with the understanding that appropriate statistical analysis is paramount, peer reviewers without statistical backgrounds can still provide the editor with invaluable feedback. For example, studies reporting multiple time points for a single measure (eg, serum tumor markers) should use statistical tests accounting for repeated measures in the analysis. If they do not, the reviewer alerts the editor, who can request a formal statistical review. For complex analyses, the author list ideally includes a biostatistician who was responsible for the analysis and involved in the conception and execution of the study methodology. Increasingly, the involvement of a biostatistician is a requirement of many manuscripts that use anything more than a descriptive analysis.

7. Institutional review board (IRB): A statement of IRB approval or exemption and informed consent (if indicated) is required and placed in the paragraph on study design or in the statistical analysis section. Even systematic literature reviews, meta-analyses, and secondary analysis of de-identified datasets require a statement that the study was reviewed by the IRB and found to be exempt.

\section{RESULTS}

The results section presents the analysis of all stated study aims and any additional hypothesis-generating analyses of interest. Study findings are not interpreted in the results section; the findings are presented objectively, allowing the reader to draw his or her own conclusions.

As a rule, the first paragraph summarizes the study population and highlights differences in baseline characteristics between any comparison groups. It is customary for the first table of a clinical paper to provide a descriptive summary of the population characteristics, stratified by comparison groups. Major differences may indicate imbalance between the 2 groups and require more advanced statistical analysis, including regression or propensity-score methods, newer instrumental variable methods, or the inclusion of a control group. One problem encountered often is the inclusion of too much baseline data, which can detract from the overall message. If the authors have chosen to include an exhaustive list, an outstanding reviewer will suggest that they focus on key variables and jettison other necessary, but less important baseline data to an appendix.

Results should proceed logically, following the order presented in the study aims and methods, beginning with the primary aim and then working through the other aim(s). It is common to see all of the data for one group presented in a paragraph, including the demographics and baseline characteristics, analysis of predictors, and then the outcomes, followed by the same details for the comparison group in another paragraph. This approach makes it hard to analyze the comparison data between the 2 groups; in this case, the reviewer should instruct the authors to reorganize the results.

\section{Tables and Figures}

Tables and figures are critically important in presenting results to the reader. As such, a careful examination of the tables and figures is vital. All tables and figures should be clearly labeled and present the results in a logical manner. The text should not duplicate the tables and figures; rather, a well-written results section should guide the reader to each table and figure at the appropriate points to maintain the logical sequence of results presentation. Numbers and 
TABLE 3. Reporting guidelines by study type ${ }^{6}$ (see permissions for use)

\begin{tabular}{ll}
\hline Randomized trials & CONSORT \\
& Consolidated Standards of Reporting Trials \\
Observational studies & STROBE \\
& Strengthening the Reporting of Observational \\
& Studies in Epidemiology \\
Systematic reviews & PRISMA \\
& Preferred Reporting Items for Systematic \\
& Reviews and Meta-Analyses \\
Diagnostic/prognostic & STARD \\
studies & Standards for Reporting of Diagnostic \\
& Accuracy \\
& TRIPOD \\
& Transparent Reporting of a Multivariable \\
& Prediction Model for Individual Prognosis \\
& or Diagnosis \\
& SRQR \\
Qualitative research & Standards for Reporting Qualitative Research \\
& COREQ \\
& Consolidated Criteria for Reporting \\
& Qualitative Research \\
& CARE \\
& Case Reports \\
Case reports & SQUIRE \\
Quality improvement & Standards for Quality Improvement \\
& Reporting Excellence \\
& CHEERS \\
& Consolidated Health Economic Evaluation \\
& Reporting Standards \\
\hline & \\
& \\
& \\
& \\
& \\
&
\end{tabular}

percentages should be properly rounded and only as many decimals points as needed to communicate the data should be used. Confidence intervals are important for understanding the precision of the data and should be reported in many situations, such as reporting of odds ratios and survival data. Continuous data should be reported as the mean and standard deviation only if they are normally distributed. If the data are not normally distributed, the median and interquartile range are used. Comparisons, likewise, should use statistical tests that account for the lack of normality. ${ }^{8}$

Figures should enhance the presentation of data and add to the reader's understanding of the analysis. The reviewer needs to determine whether the figure aids in understanding of the analysis (for example, area under the receiver operator curves) and presentation of results, and recommend removal when appropriate. Figures showing time-varying analyses, such as Kaplan-Meier curves and actuarial curves, are often missing important components, including periodic display of the confidence limits surrounding the data points, censoring events, and the number of patients at risk at each time point. These components are necessary to understand the data and meticulous reviewers advise the authors to include them in the manuscript revision.

\section{DISCUSSION}

The discussion section allows the authors to explain the study importance to the reader, and to provide clinical perspective and context. As a general rule, the first paragraph should summarize the study hypothesis, aims, and key findings. Subsequent paragraphs can be used to discuss any relevant published data. In particular, discrepant studies should be referenced and the authors should provide a rationale for disparities. The discussion should underscore what new questions their data raises and what types of future studies are suggested to explore these questions. Hypothesis generation is an important result of any well-designed study.

A paragraph describing the study limitations is also mandatory. This paragraph typically begins with a statement of the study strengths and then proceeds with a detailed list of the limitations of the data and the analysis to address the study question(s). The authors should indicate if, and how, they were able to overcome the limitations and whether or not the study is generalizable. If the study is not generalizable, the populations to which the study applies should be stated. Any limits to data interpretation should be noted.

The final paragraph reiterates the main study finding(s), concludes with the central inferences for the reader, and denotes where future research might be focused. These inferences include relevance of the study findings to the reader's clinical practice indicating why the study findings are relevant to their practice and how she or he might implement the recommended changes.

\section{SUBMITTING YOUR RECOMMENDATIONS}

Efficient reviewers are timely in submitting their recommendations and candid about telling editors and authors what is great (or not) about the manuscript. Constructive and specific feedback is key. Reviews that are too critical or inconsistent are of limited value, as are reviews that are superficial and do not suggest opportunities for improvement. Reviewers are typically asked several questions about the scientific value, originality, and analytic rigor of the study, and whether biostatistical review is needed. Most journals offer 3 categories of recommendations: (1) accept as is; (2) revision and re-review; and (3) reject. Manuscripts are rarely accepted as is; insightful reviewers can always offer the authors suggestions to strengthen a manuscript. Major and uncorrectable flaws with the study design, inadequately powered studies, or unsurmountable problems with study definitions or outcomes are potential reasons for rejection.

Most editorial sites include a "Comments to Editor" section; reviewers are encouraged to use this section to explain their recommendation to the editor. These "Comments" should reflect a clear understanding and 
accurate critique of study strengths and weaknesses. Statements should not be arbitrary or unsupported; they should provide the editor unbiased and objective justification for the recommendation.

\section{TIPS FOR IMPROVING THE QUALITY OF YOUR REVIEW}

It is common for reviewers to focus their review on the introduction and the conclusions, assuming that the authors have designed and executed a study that is appropriate for the study question. Many times, however, this is not the case, and a more critical appraisal identifies opportunities for major improvements in the manuscript. In particular, many reviewers shy away from analysis of the study methodology because of lack of expertise. There are several ways for reviewers to improve their ability to critique study design and to determine whether the analysis and presentation of results is appropriate for the study question.

1. Seek mentorship in the review process from individuals who serve on editorial boards and who have an interest in high-quality peer-review processes. Most journals allow reviewers to enlist another individual to co-review the manuscript. A good mentor will provide initial guidance and subsequent feedback about the review as well as revising the review based on his or her own critical evaluation of the manuscript.

2. Take the time to improve understanding of statistical analysis through use of the Internet and other resources to research statistical tests reported in each study.

3. Most journals provide access to other reviewer comments; it is very instructive to review other reviewer comments for the same manuscript after submitting your own review. The good reviews and the bad reviews are equally valuable. The good reviews will provide detailed critiques on study design, analysis, and presentation of results, as detailed previously, whereas the bad ones will provide little to aid the editors in their decisions and the authors in manuscript revisions.

4. Journals often provide feedback and ratings for reviewers based on timeliness and quality of reviews.
5. Collaborate with a biostatistician when writing your own papers and engage in discussions about accurate study design and analysis.

6. Investigate opportunities to earn continuing medical education credits for peer-review activities, as some journals now offer this.

By approaching editorial reviewing as a constant learning process, reviewers develop and improve with each opportunity to engage in the editorial process.

\section{CONCLUSIONS}

Consumers of medical literature expect peer-reviewed publications to be accurate, meaningful, and ideally have high impact. Meeting this expectation requires a thorough and critical evaluation of submitted manuscripts through the peer-review process. An effective peer-review system will facilitate informed and sound clinical practice changes that will enhance value-based care for our patients and iteratively educate the entire field of cardiothoracic surgery.

\section{Conflict of Interest Statement}

Authors have nothing to disclose with regard to commercial support.

\section{References}

1. Spight M, Arts ICW. How to review a manuscript. J Clin Epidemiol. 2010;63: 1385-90.

2. Kotsis SV, Chung KC. How to submit a revision and tips on being a good peer reviewer. Plast Reconstr Surg. 2014;133:958-64.

3. Hoppin FG. How i review an original scientific article. Am J Respir Crit Care Med. 2002;166:1019-23.

4. Browner WS. Introduction: Publishing and Presenting Clinical Research. 2nd ed. Philadelphia: Lippincott Williams \& Wilkins; 2006:21-6.

5. Hulley SB, Cummings SR, Browner WS, Grady DG, Newman TB. Getting Started: The Anatomy and Physiology of Clinical Research: Designing Clinical Research. 3rd ed. Philadelphia: Lippincott Williams \& Wilkins; 2007:3-15.

6. Equator Network. Enhancing the quality and transparency of health research. Available at: http://www.equator-network.org/. Accessed September 25, 2016.

7. CONSORT. Transparent reporting of trials. Available at: http://www.consortstatement.org/consort-statement/flow-diagram. Accessed September 25, 2016.

8. Katz MH. Bivariate Statistics: Study Design and Statistical Analysis: A Practical Guide for Clinicians. New York: Cambridge University Press; 2006:66-119.

9. Collins FS, Tabak LA. Policy: NIH plans to enhance reproducibility. Nature. 2014;505:612-3.

10. Katz MH. Setting Up a Multivariable Analysis: Multivariable Analysis: A Practical Guide for Clinicians. 2nd ed. New York: Cambridge University Press; 2006: 73-95. 